\title{
Compliant Control for Walking Robots with the Use of a Virtual Spring-Damper System
}

\author{
S. Golousov，R. Khusainov，S. Savin
}

The paper deals with one of the modern challenges in walking robotics: moving across a rough terrain where the geometry of the terrain is unknown and hence it is impossible to plan precise trajectories for the robot feet in advance, before a collision with the supporting surface occurs. In this paper, an algorithm for the dynamics correction of the foot trajectory based on the compliant control is employed to deal with the problem. Additionally, to solve the problem of dynamic correction of the foot trajectory, it also provides a biomorphic reaction force profile, which might be a desired property for some applications.

Keywords: walking robot, uneven terrain, compliant control, biomorphic reaction force profile

\section{Introduction}

Walking over a rough terrain is an interesting and challenging topic in modern humanoid robotics $[1,2]$. Let us consider the problem of controlling a bipedal robot walking on a terrain with an unknown geometry. This scenario might be a direct consequence of the limited accuracy of the sensor data and of the algorithms that use that data to produce maps of the supporting surface; which makes the otherwise deterministic surface unknown. It can also be the property of the surface itself, such as the case with the surfaces covered by grass and other objects that

Received May 15, 2019

Accepted July 22, 2019

This work has been supported by the Ministry of Science and Higher Education of the Russian Federation with the project "Development of Anthropomorphic Robotic Complexes with Variable Stiffness Actuators for Movement on the Flat and Rugged Terrains" (agreement: No. 075-10-2019-049 (No.14.606.21.0007), ID: RFMEFI60617X0007).




do not provide support but hide geometrical features of the terrain. To a certain degree, limited knowledge of the surroundings is a natural state for mobile robots in general. This makes the topic of controlling a walking robot interesting regardless of the quality and quantity of sensors that are available to the particular robot.

One of the problems with walking on the terrain with unknown geometry is that it is possible that the contact scenario (the number of contacts, the position of each of them and the local tangent plane to the supporting surface for each of the contacts) might change unpredictably, or change at a different time instant compared to when it was planned to have changed. For example, a foot of the robot can acquire or lose contact with the supporting surface before it was supposed to have happened. Untimely acquisition of the contact can lead to a collision, while untimely loss of contact can lead to a significant increase in the error in the Cartesian space for the points of interest of the robot.

One of the ways of solving this problem is the use of the compliant control [3]. The compliant control here refers to the control paradigm aiming at making the dynamical system, formed by the controlled object and its controller, to act as a mechanical system with natural compliance, such as a spring-dumper system. This control paradigm is especially focused on the interactions with the environment and external agents. One of the famous examples of the compliant control is the control of collaborative robots arms, where the controller allows the operator to freely grasp and move the end effector of the manipulator, exerting very limited forces on the robot. The robot arm meanwhile can hold its position or imitate a linear oscillator (a linear dynamical system made with a spring, a damper and a mass) that tries to return to the initial position in the absence of external forces. Compliant control is also used in the feedback channels for the master-slave systems and teleoperation control systems, where a human operator is involved, as well in other areas related to the cooperative robotics [4].

When using compliant control in walking robots, the aim is to implement the trajectory control for the robot's pedipulators (its feet) in such a way as to allow them to dynamically react to the collisions with the supporting surface which can occur at an unpredicted time. The reaction comes in the form of a change in the joint torques, taking into account the information of the currently observed reaction forces.

\section{Compliant control with the use of a virtual spring-damper system}

Compliant control of walking robots, especially of ones with elastic or various stiffness actuators (VSA), introduces a problem of great interest. In [5] a compliant control synthesis approach for walking robots is suggested. It incorporates using of a virtual spring-damper system, which describes simplified dynamics of the point at the end of the robot leg. The equation of such a dynamical system could be formulated in the following way:

$$
M\left(\ddot{z}^{*}-\ddot{z}\right)+K_{d}\left(\dot{z}^{*}-\dot{z}\right)+K_{p}\left(z^{*}-z\right)=f^{*}-f
$$

where $M$ is the inertia matrix of the virtual system, $K_{d}$ and $K_{p}$ are the viscous friction and stiffness coefficients of the virtual system, $f^{*}$ and $f$ are the desired and real values of ground reaction forces, and $z^{*}$ and $z$ are the desired and real position of the leg point in projection on the contact surface normal.

Equation (2.1) could be used to find the desired trajectory $z=z(t)$ in Cartesian space. Then the joint space trajectory could be found as the time function $\mathbf{q}=\mathbf{q}(t)$ from the Carte- 
sian trajectory solving inverse kinematics. This allows us to use conventional feedback control algorithms for tracking the desired trajectories $z=z(t)$.

The suggested algorithm for compliant control could be summarized as follows. When contact force is different from the desired one, the linear system described by Eq. (2.1) is used to recalculate the robot leg trajectory in Cartesian space such that it will provide the desired contact force. Thus, Eq. (2.1) is used for dynamic correction of the robot trajectories in Cartesian space.

\section{Numeric experiments with stepping onto an obstacle with unknown height}

In this work, we consider a walking robot with two 5-DoF legs. Each leg has two joints at hip, one joint at knee and two joints at ankle, with all joints being revolute and actuated. At the beginning of the experiment the robot is statically balanced and is standing in a single support stance (initial position). Then the robot is commanded to move its swing leg vertically down. The control system has no information about the supporting surface.

Let us find the desired swing leg trajectory that implements leg movement from the initial position to the final position - 0 meters above the ground. At the same time we put an obstacle between the initial and final position at the height of 0.1 meters, such that the desired trajectory is now infeasible.

A precomputed swing leg trajectory for joint space is presented in Fig. 1. These time functions were corrected, as described above, during leg movement in response to the collision that has occurred. The corrected swing leg trajectory is shown in Fig. 2.

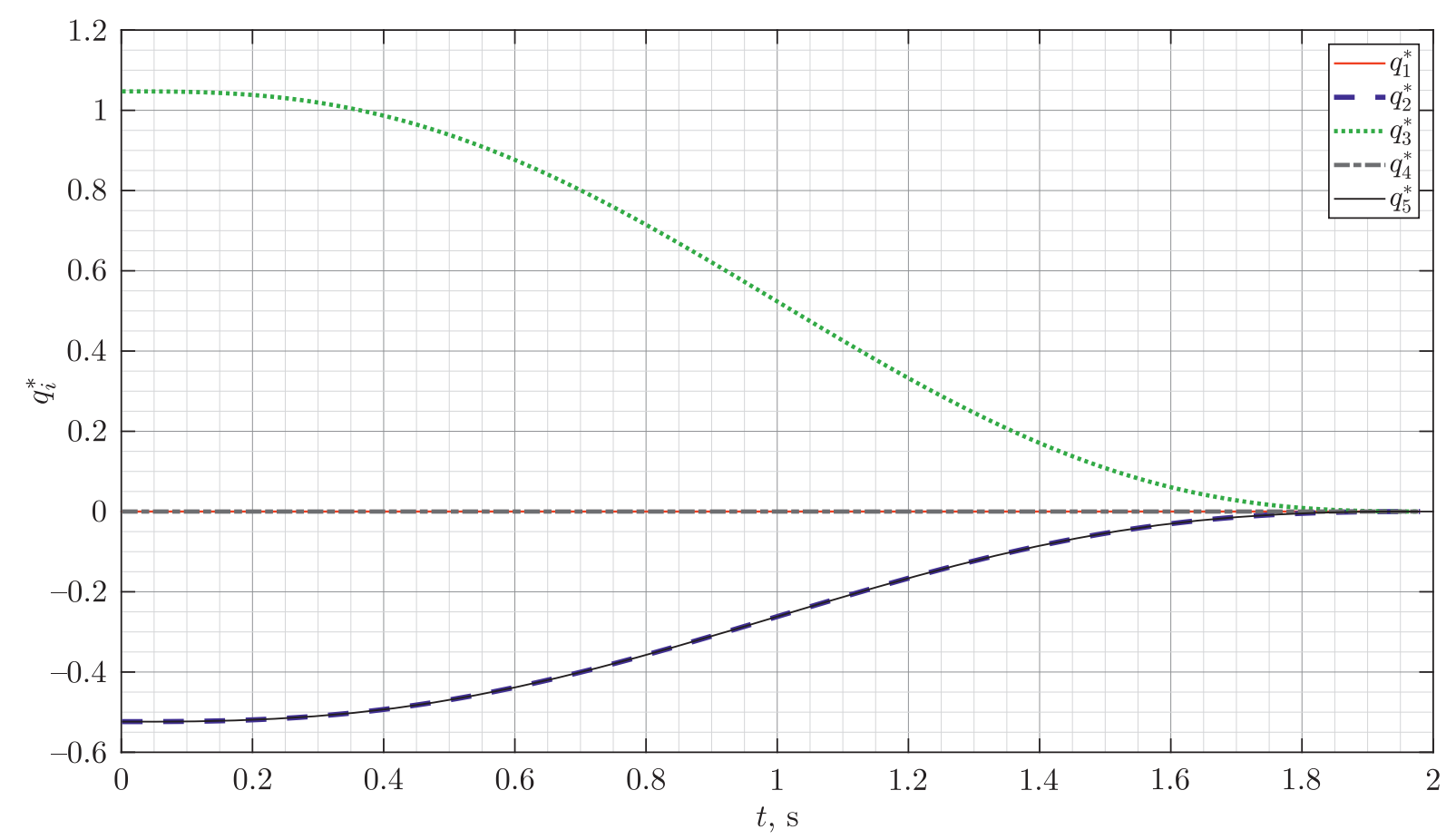

Fig. 1. Generalized coordinates trajectory as time functions for the precomputed swing leg movement. 


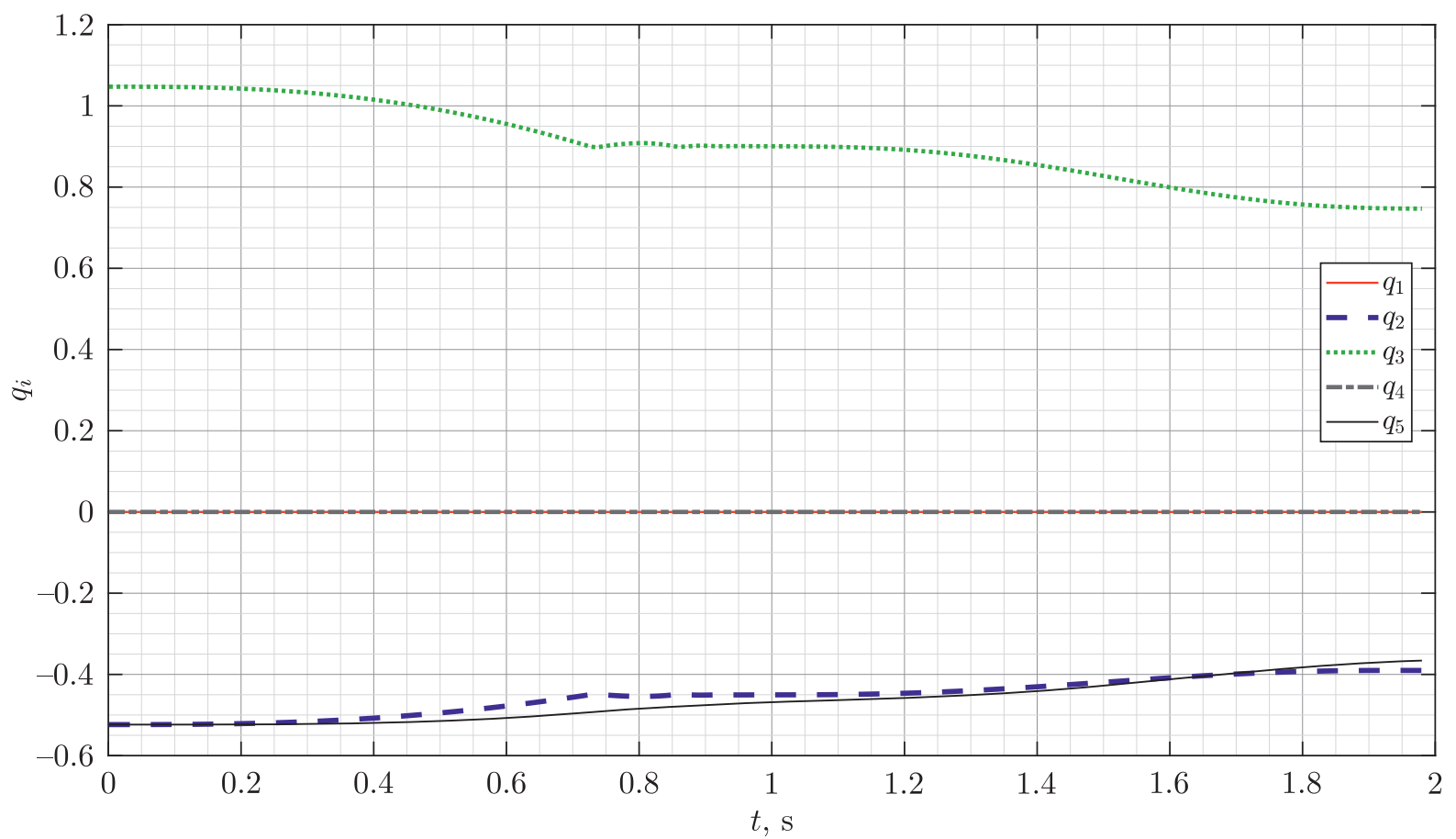

Fig. 2. Generalized coordinates trajectory as time functions for swing leg movement corrected via the compliant control approach.

We can see that the corrected trajectories seriously deviate from precomputed ones. This illustrates how the compliant control approach works: it successfully manages to correct the infeasible trajectory in response to a collision with the contact surface.

Figure 3 shows the desired and observed contact forces occurring at the swing leg during movement. There is a small peak at the collision time in the observed contact force. It agrees with similar results obtained in [5]. This implies that the results were replicated in a correct way and demonstrates drawbacks of proposed method.

The above experiments were conducted with control parameters being fixed as: $M=1$, $K_{d}=1, K_{p}=100$. In the next section, we investigate the influence of these parameters on the resulting control system performance.

\section{Study of the influence of the control system parameters on the motion characteristics of the robot}

Let us analyze how the control parameter $K_{p}$ influences the robot movement. For this we fix $M=1, K_{d}=0.01$ and vary $K_{p}$. Figures 4 and 5 present simulation results for $K_{p}$ equal to 5,50,200, 1000 and 5000. Figure 4 shows the desired and observed normal contact forces during robot movement, while Fig. 5 depicts the swing leg position projected on the contact surface normal vector.

One can observe that for $K_{p}$ equal to 5 and 50, the graphs in Fig. 4 coincide. The same holds for $K_{p}$ equal to 1000 and 5000 .

We should note that the increase in $K_{p}$ affects the accuracy of the observed contact forces $f^{*}=f^{*}(t)$. At the same time, low $K_{p}$ values cause the swing leg to "bounce" from the contact 


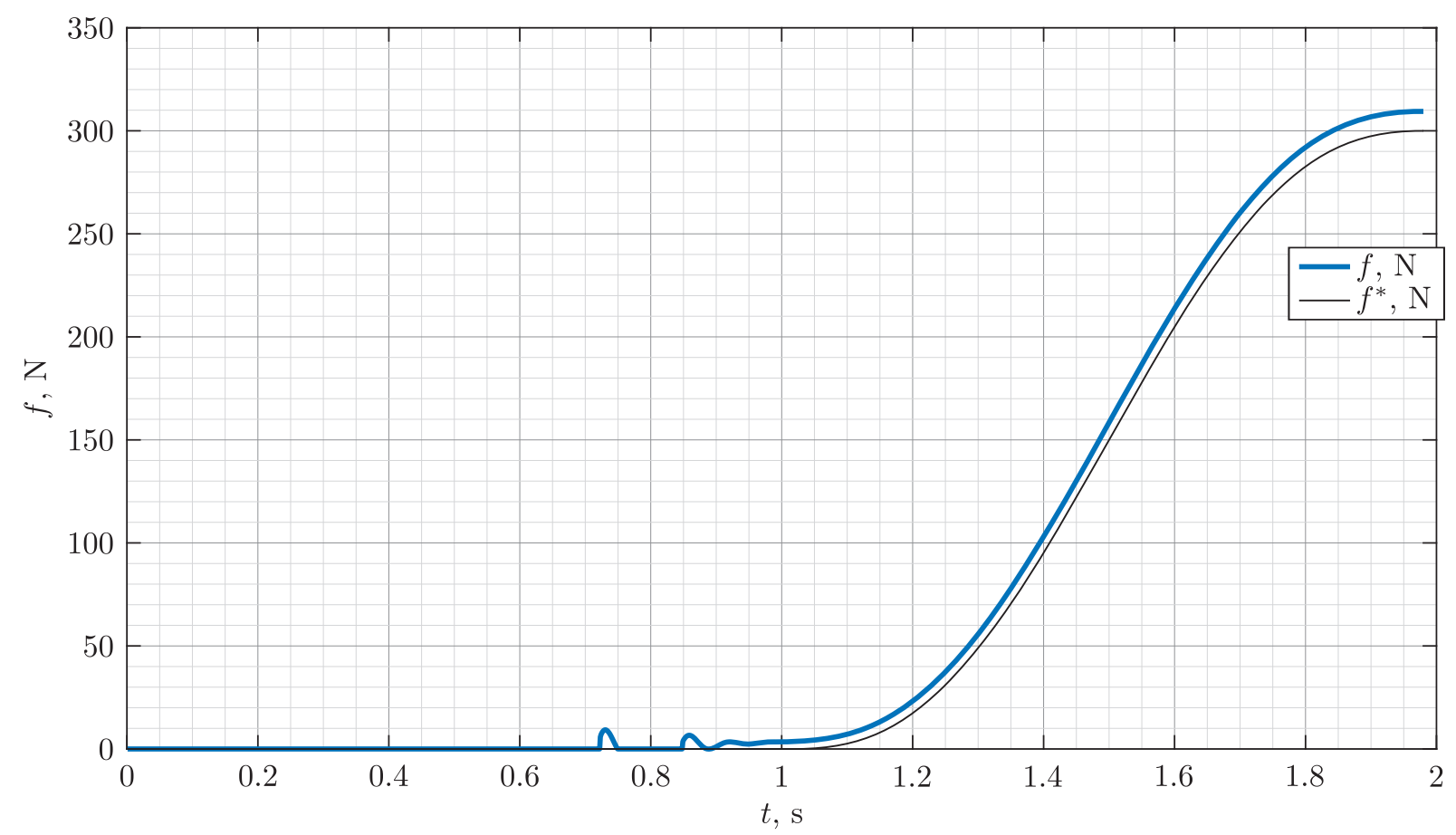

Fig. 3. Normal contact forces with respect to time.

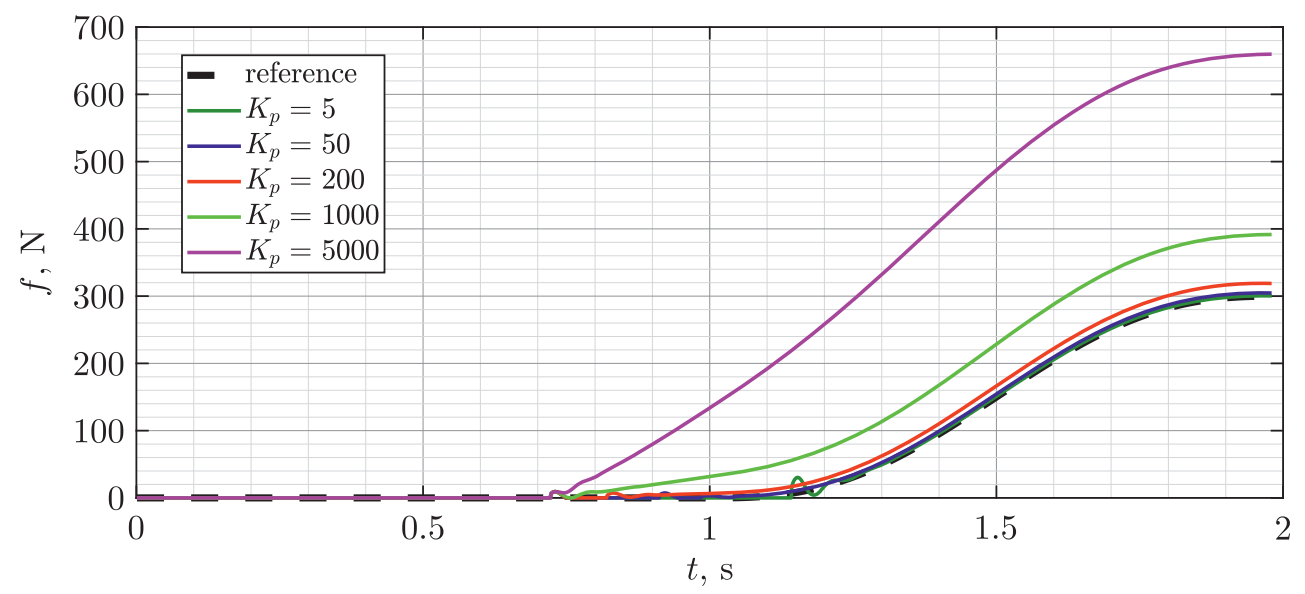

Fig. 4. Normal contact forces with respect to time for various $K_{p}$ coefficients.

surface in response to the collision. Thus, when using the compliant control approach described in this paper these two negative effects should be taken into account.

Next, we investigate how the coefficient $K_{d}$ influences the motion of the robot. For this, we fix $M$ at $M=1, K_{p}=100$ and conduct experiments for $K_{d}$ equal to $10,100,200,500$ and 1000. Figure 6 shows time functions of the desired and observed contact forces during the robot movement.

Figure 6 shows that the increase of the coefficient $K_{d}$ leads to an increase in contact force tracking error. Thus, we need to use the smallest possible coefficient $K_{d}$, such that the observed functions $f=f(t)$ do not oscillate, if the absence of oscillation is important to achieve the required quality of robot movement. 


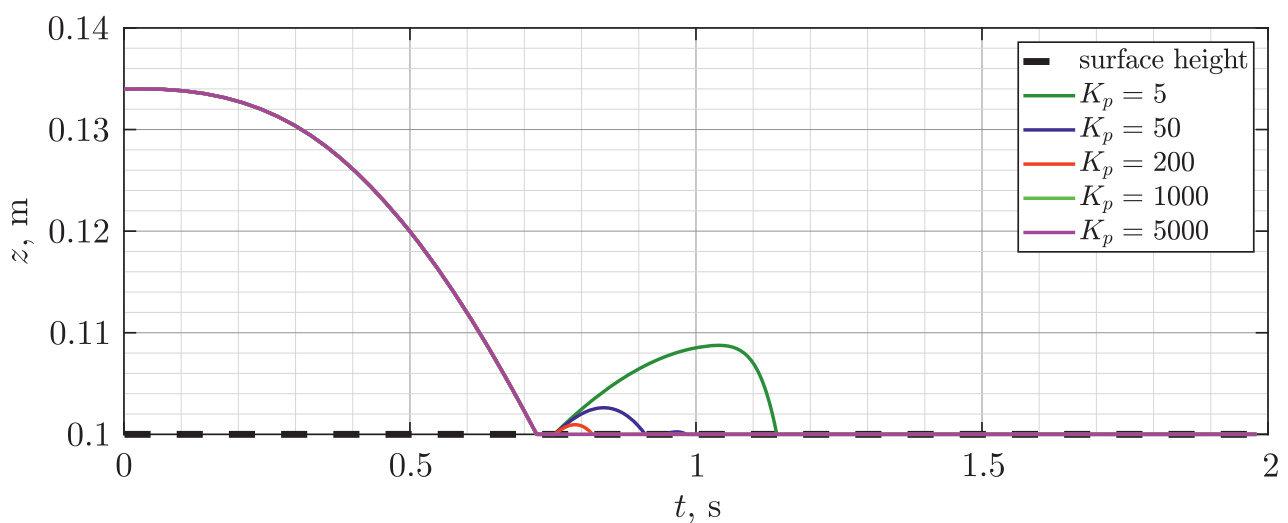

Fig. 5. Swing leg position projected on the contact surface normal vector for various $K_{p}$ coefficients.

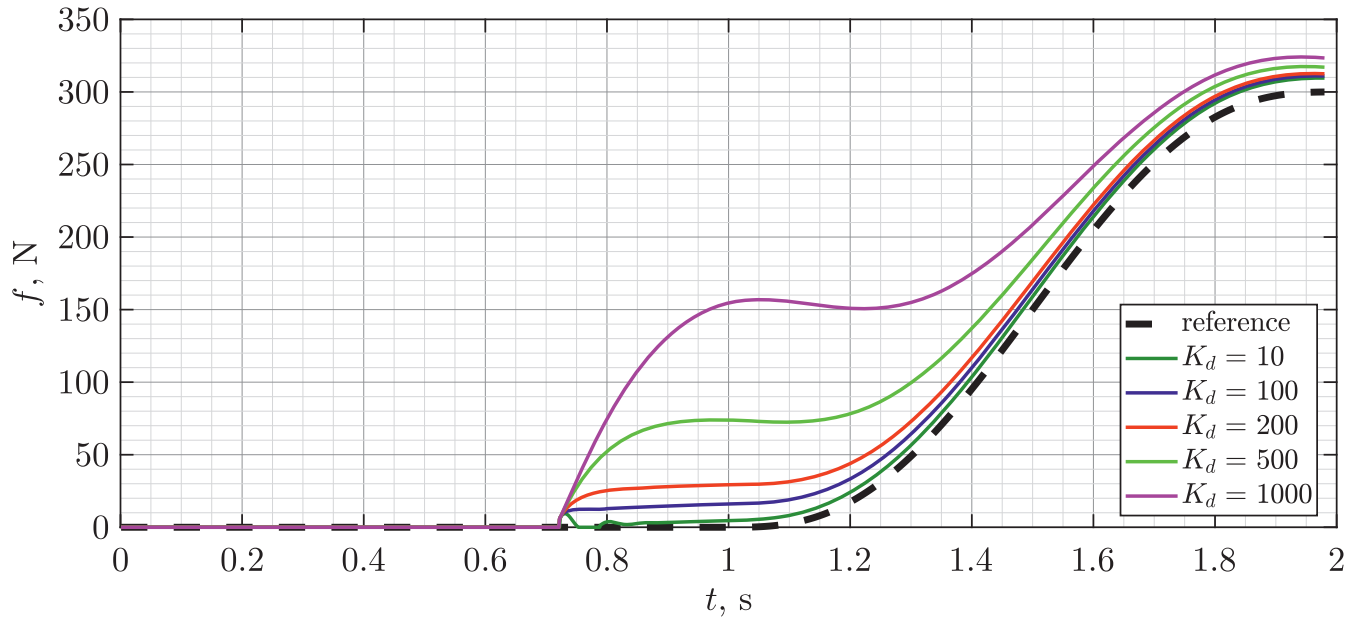

Fig. 6. Normal contact forces with respect to time for various $K_{d}$ coefficients.

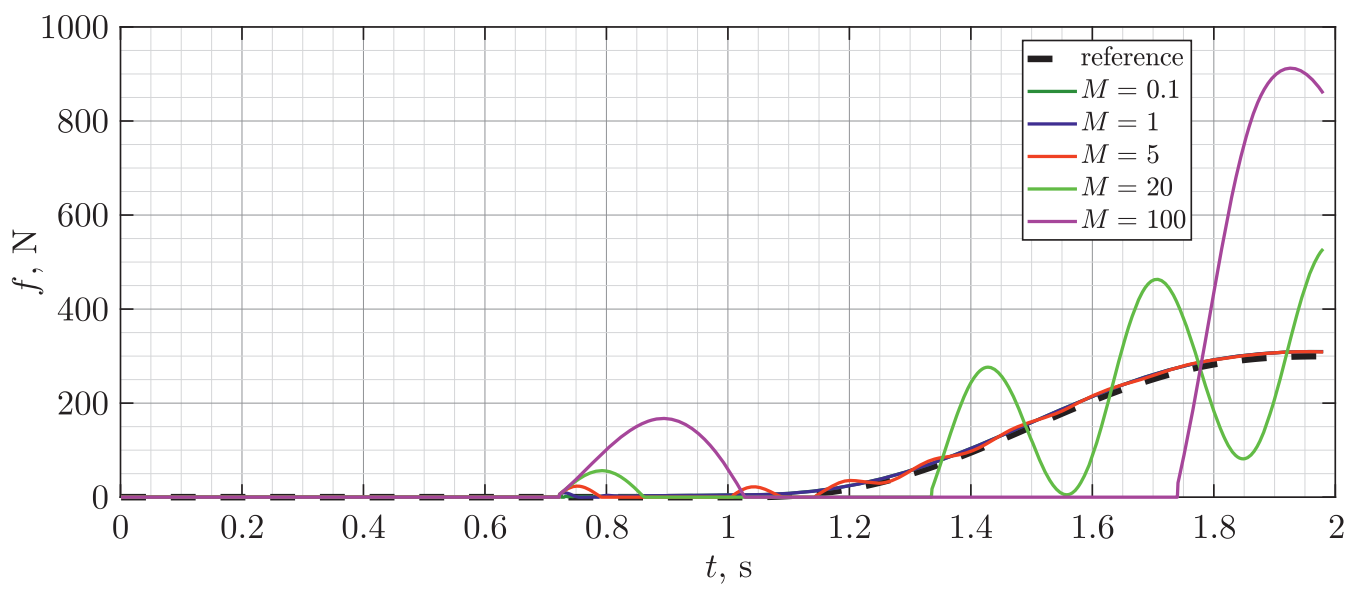

Fig. 7. Normal contact forces with respect to time for various $M$ coefficients. 


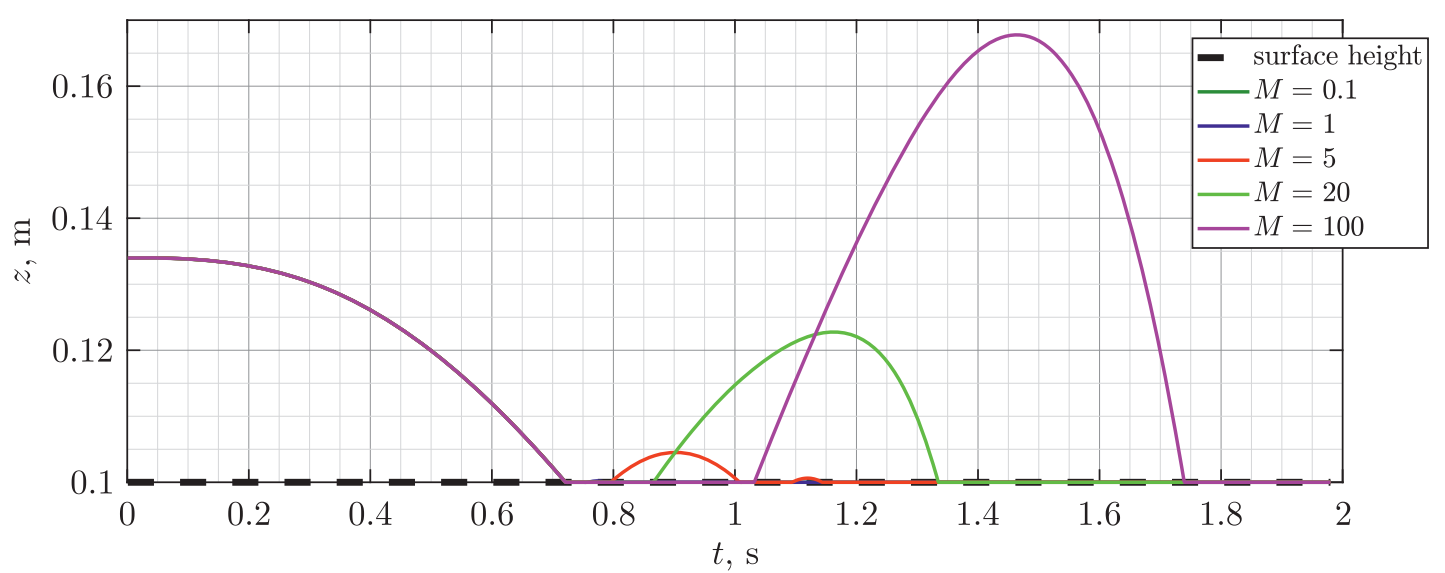

Fig. 8. Swing leg position projected on the contact surface normal vector for various $M$ coefficients.

Finally, let us fix $K_{p}=100$ and $K_{d}=10$ and analyze how different $M$ values affect the robot movement. For this we take $M$ to be equal to 0.1, 1, 5, 20 and 100. Figure 7 shows desired and observed normal contact forces during the movement. Figure 8 depicts the changes in the leg trajectory in response to $M$ variation.

As can be seen from Fig. 7, for $M$ equal to 0.1 and 1 the contact forces trajectories almost coincide with the reference. At the same time, Fig. 8 indicates that all leg trajectories before collision coincide with each other, but after the collision only the graphs for $M$ equal to 0.1 and 1 move along the dashed line, while others bounce away.

It can be noticed that, as the inertial coefficient $M$ increases, the contact force tracking error degrades dramatically as does the performance of the leg position projected on the contact normal. From this point of view, choosing a small value for the inertial coefficient $M$ can be optimal. Similar results can be obtained from the admittance control approach [6].

\section{An implementation of a biomorphic profile of the contact forces for a bipedal walking robot with and without SEA}

In [5] a biomorphic profile of the contact forces emerging during the walking process was analyzed. This profile is characterized by two peaks in single support phase and monotonous increase and decrease in double support phases of walking. In [5] the reaction forces were switched between $0 \mathrm{~N}, 170 \mathrm{~N}, 180 \mathrm{~N}, 160 \mathrm{~N}, 180 \mathrm{~N}, 170 \mathrm{~N}$ and $0 \mathrm{~N}$ values. Figure 9 shows the desired and observed contact forces for the biomorphic force profile.

The suggested force profile was generated with the use of polynomial splines. Additional conditions for points $t=0.4 \mathrm{~s}, t=0.6 \mathrm{~s}, t=0.7 \mathrm{~s}, t=0.8 \mathrm{~s}, t=1 \mathrm{~s}$ were required: the first and second derivatives of polynomials at those points were set to zero. For points $t=0.5 \mathrm{~s}, t=0.9 \mathrm{~s}$ no conditions for derivatives were added. There are peaks at $t=0.36 \mathrm{~s}$ (at the moments of collision with the obstacle), $t=0.4 \mathrm{~s}$ (where the desired contact force becomes positive) and $t=0.5 \mathrm{~s}$ (where the derivative of the desired contact force is discontinuous).

Let us show that similar contact force profiles can be acquired for the robot with computed torque control (CTC) in combination with series elastic actuators (SEA). The general form of a CTC regulator can be written as

$$
\mathbf{u}=\mathbf{B}^{+}\left(\mathbf{H}\left(\ddot{\mathbf{q}}^{*}+\mathbf{K}_{d} \dot{e}+\mathbf{K}_{p} \mathbf{e}\right)+\mathbf{c}\right)
$$






Fig. 9. Desired and observed normal contact forces with respect to time for biomorphic force profile.

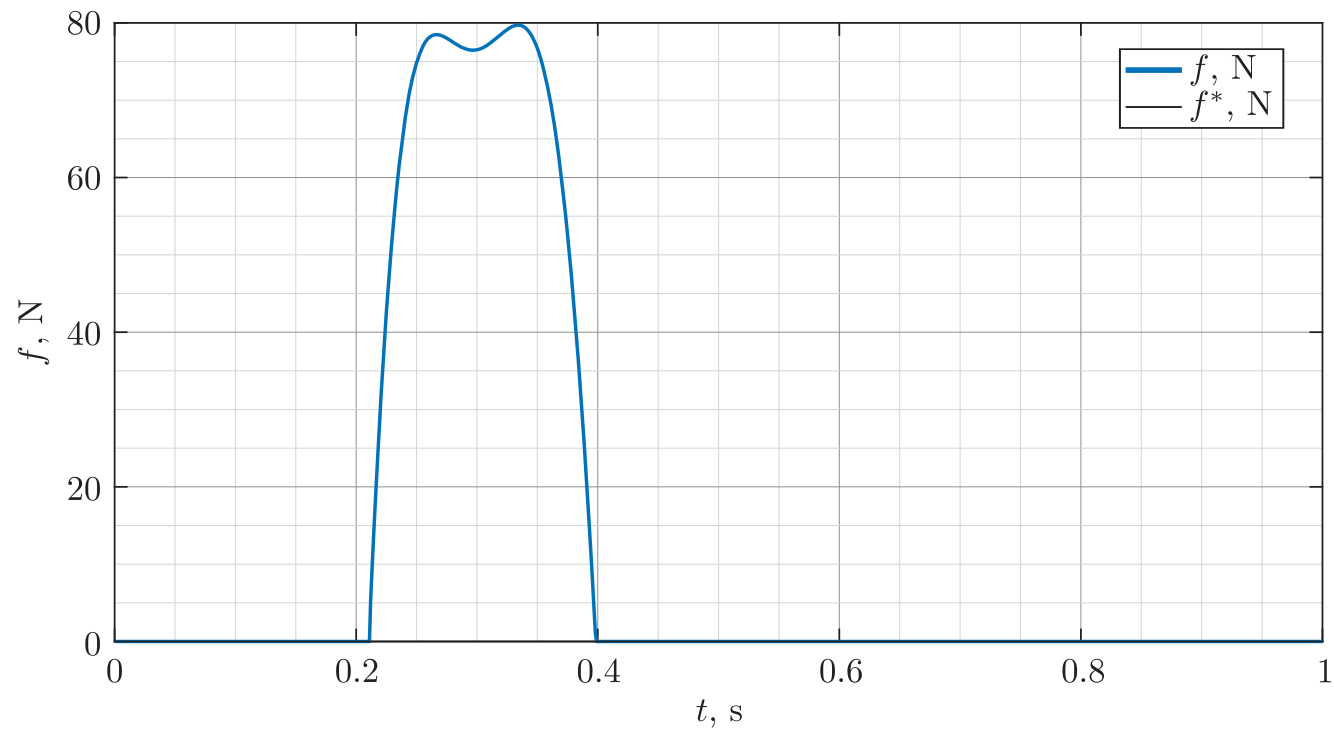

Fig. 10. Desired and observed contact forces as time functions during movement of the robot with SEA actuators.

where $\mathbf{H}$ is the generalized matrix of inertia of the robot, $\mathbf{q}$ is the vector of generalized robot coordinates, $\mathbf{c}$ is the vector of the sum of generalized inertial, gravitational and dissipative forces, $\mathbf{B}$ is the control matrix linearly transforming the actuators torques to generalized torques, $\mathbf{u}$ is the vector of actuators torques, and $\mathbf{K}_{d}$ and $\mathbf{K}_{d}$ are the positive definite gain matrices.

The SEA actuator dynamics can be formulated as follows:

$$
\left\{\begin{array}{l}
\mathbf{H}^{s} \ddot{\phi}^{s}=\mathbf{u}-\mathbf{u}^{s} \\
\mathbf{u}^{s}=\mathbf{K}_{p}^{s}\left(\phi^{s}-\phi\right)+\mathbf{K}_{d}^{s}\left(\dot{\phi}^{s}-\dot{\phi}\right)
\end{array}\right.
$$

where $\mathbf{H}^{s}$ is the SEA matrix of inertia, $\mathbf{u}^{s}$ are the moments generated by SEA spring elements, $\mathbf{K}_{p}^{s}$ and $\mathbf{K}_{d}^{s}$ are the diagonal matrices of SEA damping and dissipative properties, and $\phi^{s}$ is the vector of SEA output shafts positions.

Figure 10 illustrates computed contact forces. 
It should be noted that the observed contact force profile is biomorphic, even though the control law includes no information about the desired contact force profile. This can be the result of the robot leg having a biomorphic structure. Similar results can be obtained using various stiffness actuators which are able to change $\mathbf{K}_{p}^{s}$ during robot motion.

\section{Conclusions}

In this paper we have considered the problem of implementing a robot walking algorithm for the case where the robot moves across an uneven terrain with unknown geometry. The employed algorithm aims at dynamically changing the Cartesian trajectory of the robot foot. The algorithm manages to correct the trajectory for the case where the contact interaction occurs at a different place and at a different time compared with the reference. The paper demonstrates the properties of the algorithm based on the different choices of parameters.

\section{References}

[1] Zheng, Y., Lin, M. C., Manocha, D., Adiwahono, A. H., and Chew, Ch.-M., A Walking Pattern Generator for Biped Robots on Uneven Terrains, in Proc. of the IEEE/RSJ Internat. Conf. on Intelligent Robots and Systems (Taipei, Taiwan, 2010), pp. 4483-4488.

[2] Kanoulas, D., Stumpf, A., Raghavan, V.S., Zhou, Ch., Toumpa, A., von Stryk, O., Caldwell, D. G., and Tsagarakis, N. G., Footstep Planning in Rough Terrain for Bipedal Robots Using Curved Contact Patches, in Proc. of the IEEE Internat. Conf. on Robotics and Automation (ICRA, Brisbane, 2018), 9 pp.

[3] Sentis, L., Park, J., and Khatib, O., Compliant Control of Multicontact and Center-of-Mass Behaviors in Humanoid Robots, IEEE Trans. on Robotics, 2010, vol. 26, no. 3, pp. 483-501.

[4] Arcara, P. and Melchiorri, C., Control Schemes for Teleoperation with Time Delay: A Comparative Study, Robot. Auton. Syst., 2002, vol. 38, no. 1, pp. 49-64.

[5] Son, B. G., Kim, J. T., and Park, J.H., Impedance Control for Biped Robot Walking on Uneven Terrain, in Proc. of the IEEE Internat. Conf. on Robotics and Biomimetics (ROBIO, Guilin, 2009), pp. 239-244.

[6] Ott, Ch., Mukherjee, R., and Nakamura, Y., Unified Impedance and Admittance Control, in Proc. of the IEEE Internat. Conf. on Robotics and Automation (Anchorage, Alaska, 2010), pp. 554-561. 